\title{
Mesenchymal Stem Cell-derived Extracellular Vesicles Transmitting MicroRNA-34a-5p Suppress Tumorigenesis of Colorectal Cancer Through c-MYC/DNMT3a/PTEN Axis
}

\author{
Jiangning Zhao ${ }^{1,2}$ (D) Huanrong Lin $^{1,2} \cdot$ Kunsong Huang ${ }^{3}$
}

Received: 17 January 2021 / Accepted: 12 May 2021 / Published online: 8 October 2021

(C) The Author(s) 2021

\begin{abstract}
Mesenchymal stem cell-derived extracellular vesicles (MSC-EV) can transport microRNAs (miRNAs) into colorectal cancer (CRC) cells, thus to inhibit the malignant phenotype of cancer cells. Whether MSC-EV could deliver miR-34a-5p to suppress CRC development was surveyed through the research. miR-34a-5p, c-MYC, DNA methyltransferase 3a (DNMT3a), and phosphatase and tensin homolog deleted on chromosome 10 (PTEN) expression were measured in CRC tissues and cell lines. miR-34a-5p and c-MYC expression were altered by transfection in HCT-116 cells. MSC-EV were transfected with miR-34a-5pand c-MYC-related oligonucleotides and co-cultured with HCT-116 cells. HCT-116 cell growth after treatment was observed. Furthermore, the functional roles of miR-34a-5p and c-MYC were explored in vivo. The combined interactions of miR-34a-5p/cMYC/DNMT3a/PTEN axis were assessed. miR-34a-5p and PTEN were downregulated while c-MYC and DNMT3a were upregulated in CRC. Depletion of miR-34a-5p drove while that of c-MYC restricted CRC cell growth. MSC-EV retarded CRC progression. Moreover, MSC-EV carrying overexpressed miR-34a-5p or depleted c-MYC further disrupted CRC cell progression. miR-34a-5p targeted c-MYC to regulate DNMT3a and PTEN. c-MYC overexpression abrogated EV-derived miR-34a-5p upregulation-induced effects on CRC. Restoring miR-34a-5p or depleting c-MYC in MSC-EV limited CRC tumor formation. MSC-EV-derived miR-34a-5p depresses CRC development through modulating the binding of c-MYC to DNMT3a and epigenetically regulating PTEN.
\end{abstract}

Keywords Colorectal cancer - Mesenchymal stem cells $\cdot$ Extracellular vesicles $\cdot$ MicroRNA-34a-5p $\cdot$ c-MYC $\cdot$ DNA methyltransferase $3 \mathrm{a} \cdot$ Phosphatase and tensin homolog deleted on chromosome 10

\section{Introduction}

Colorectal cancer $(\mathrm{CRC})$ is a heterogeneous tumor presenting non-uniform molecular patterns that is stemmed from nonmalignant adenomas [1]. Red and processed meat intake, alcohol abuse, obesity, inflammatory bowel disease, and family history of CRC consist of the risk factors for CRC [2]. CRC

Jiangning Zhao

Zhaojiangning598@outlook.com

1 Gastrointestinal Peritoneal Cancer Surgery, The Fourth Clinical Medical School of Guangzhou University of Chinese Medicine, 1 Fuhua Road, Futian District, Shenzhen 518033, Guangdong, China

2 Shenzhen Traditional Chinese Medicine Hospital, 1 Fuhua Road, Futian District, Shenzhen 518033, Guangdong, China

3 Department of General Surgery, Guangzhou Overseas Chinese Hospital, The First Affiliated Hospital of Jinan University, Guangzhou, Guangdong, China has atypical clinical symptoms in its early stages, leading to delayed diagnosis and treatment [3]. Clinically, since the 5year survival rate of metastatic CRC is much more lower than that of CRC in stage I, promising targeted drug therapies have been developed for metastatic disease [4]. Moreover, omics technologies including metabolomics, transcriptomics, gnomics, and proteomics are utilized to improve diagnosis and treatment of CRC [5]. Thus, developments in targeted agents and armamentarium are of great importance to improve survival of CRC.

Extracellular vesicles (EV) are cell-derived membranous structures including exosomes and microvesicles [6]. Mesenchymal stem cell-derived exosomes are therapeutic tool in regenerative medicine [7] and develop an emerging strategy for CRC therapy due to their actions in growth and metastasis of cancer cells [8]. Secreted by tumor cells, exosomes contain various biomolecules that may induce angiogenesis [9] and CRC-derived exosomes have promising therapeutic value for CRC diagnosis [10]. Pharmacological 
approaches, such as drug encapsulation with exosomes are assumed to improve CRC treatment [11]. MSC-derived exosomes encapsulation has been once practiced with doxorubicin that offers a versatile platform to fight against $\mathrm{CRC}$ [12]. It is noted that bone marrow MSC-derived exosomes can deliver microRNAs (miRNAs) to restrict the tumorigenicity of CRC [13]. Dysregulated miR-34a-5p has been mentioned in CRC physiopathology [14]. miR-34a-5p has predictive value for recurrence and metastasis of CRC patients in stage II/III [15]. In fact, CpG-methylation of miR-34a is the mediated actor in primary CRC, as to cell drug resistance, and the processes of epithelial-mesenchymal transition (EMT) and metastasis [16]. Having ability to interact with miR-34a-5p [17], $\mathrm{c}-\mathrm{MYC}$ is a proto-oncogene whose therapeutic inhibitor has been discovered to protect against CRC [18]. c-MYC stability is essential to regulate colon cancer (CC) cell survival [19], as well as invasive phenotype of CRC tumors [20]. c-MYC collaborates physically with DNA methyltransferase 3a (DNMT3a) [21] that is overexpressed in para-carcinoma tissues of sporadic CRC [22]. It is worthy that low doses of DNMT inhibitors can block the development of colitisassociated cancer [23]. DNMT3a can mediate epigenetic silencing of phosphatase and tensin homolog deleted on chromosome 10 (PTEN) [24] which is a mediator for the biological functions of CC cells $[25,26]$. Based on those reports, this study aimed at elucidating the potential mechanism of miR34a-5p/c-MYC/DNMT3a/PTEN axis in CRC.

\section{Methods and Materials}

\section{Ethics Statement}

The research protocol was approved by the ethics committee of The Fourth Clinical Medical School of Guangzhou University of Chinese Medicine; Shenzhen Traditional Chinese Medicine Hospital, and written informed consent was obtained. All animal experiments were conducted with the guidelines for the care and use of laboratory animals of the National Institutes of Health.

\section{Samples}

CRC tissue and para-cancer normal tissue samples were obtained from The Fourth Clinical Medical School of Guangzhou University of Chinese Medicine; Shenzhen Traditional Chinese Medicine Hospital. All the 85 pairs of tissues were obtained through surgery, frozen, and stored in liquid nitrogen [27]. The clinicopathological characteristics of the patients were shown in Table 1.
Table 1 Clinicopathological characteristics of CRC patients

\begin{tabular}{lll}
\hline Clinicopathological characteristics & Cases $(n=85)$ & Percent $(\%)$ \\
\hline Age (years) & & \\
$\leq 60$ & 45 & $52.90 \%$ \\
$>60$ & 40 & $47.10 \%$ \\
Gender & & \\
Male & 47 & $55.30 \%$ \\
Female & 38 & $44.70 \%$ \\
Tumor stage & & \\
I-II & 36 & $42.40 \%$ \\
III & 49 & $57.60 \%$ \\
Hereditary cancer & & \\
Yes & 21 & $24.70 \%$ \\
No & 64 & $75.30 \%$ \\
Genetic screening & & \\
Yes & 32 & $37.60 \%$ \\
No & 53 & $62.40 \%$ \\
\hline
\end{tabular}

\section{Cell Culture and Transfection}

CRC cell lines HCT-116, SW-480, LoVo, and normal cell line HEK293 were acquired from ATCC (Rockville, MD, USA). Leibovitz's L-15 medium was adopted for SW-480 cell culture, Ham's F12K medium for HCT-116 and LoVo cell culture, and Dulbecco's modified eagle medium (DMEM) for HEK293 cell culture. All the culture medium contained 10\% fetal bovine serum (FBS).

miR-34a-5p- and c-MYC-related oligonucleotides sequences were provided by Sangon (Shanghai, China). The oligonucleotides were transfected into HCT-116 cells using Lipofectamine 3000 (Invitrogen, CA, USA), including negative control (NC)-inhibitor, miR-34a-5p-inhibitor, si-c-MYC and si-NC. Phosphate-buffered saline (PBS) was utilized as a NC [28].

\section{Extraction of EV}

Bone marrow was mixed with MesenPRO RS medium (12746-012, Gibco, CA, USA) and separated using h-BMMSC Isolation Kit (TBD). The collected cells were cultured with the medium changed every 2-3 days. Confluent monolayers of cells were observed 7 days later.

MSCs at $80 \%$ confluence were washed twice with PBS, and cultured in EV-free $10 \%$ FBS medium for $48 \mathrm{~h}$. Then, the supernatant was centrifuged at $500 \mathrm{~g}$ for $15 \mathrm{~min}$ to remove cell debris, at $2000 \mathrm{~g}$ for $15 \mathrm{~min}$ to remove cell debris or apoptotic bodies, and at 10,000 $\mathrm{g}$ for $20 \mathrm{~min}$ to remove large vesicles. After filtering with a 0.22 micron filter, the sample was centrifuged at $110,000 \mathrm{~g}$ for $70 \mathrm{~min}$ at $4{ }^{\circ} \mathrm{C}$, resuspended in PBS and ultra-centrifuged at $110,000 \mathrm{~g}$ for $70 \mathrm{~min}$ at $4{ }^{\circ} \mathrm{C}$ [29]. 


\section{Identification of EV}

EV were fixed with $2 \%$ phosphotungstic acid at $4{ }^{\circ} \mathrm{C}$, airdried, observed under a TEM at $80 \mathrm{kV}$ (FEI Tecnai G2 Spirit, Thermo Scientific, USA) and analyzed by western blot [30].

\section{Internalization of EV}

EV and PKH26 $(4 \mu \mathrm{L})$ were resuspended in Diluent $\mathrm{C}(1 \mathrm{~mL})$, respectively. The EV suspension was mixed with the staining solution and added with an equal volume of $1 \%$ BSA. The labeled exosomes were ultracentrifuged at $100,000 \times g$ for 70 min and then ultracentrifuged again [31]. The PKH26labeled EV were resuspended in $100 \mu \mathrm{L}$ PBS.

HCT116 cells seeded on a 24 -well plate at $1 \times 10^{6}$ cells/ well were routinely cultured for $12 \mathrm{~h}$, and added with PKH26labeled EV (10 $\mu \mathrm{g} /$ well) for 48 -h reaction. The cells were fixed with $4 \%$ paraformaldehyde, stained with 4',6diamidino-2-phenylindole and observed under a fluorescence microscope. Red fluorescence represented the uptake of PKH26-labeled EV [32].

\section{Cells Co-culture with EV}

HCT-116 and MSC were detached with trypsin, centrifuged at $1000 \mathrm{~g}$, and then resuspended in DMEM ( $3 \mathrm{~mL})$. Next, the suspension $(1 \mathrm{~mL})$ was diluted 20 times and counted. HCT116 and MSC were spread over a co-culture chamber $(0.4$ $\mathrm{mm}$ ) at 3:1. MSC (about $4 \times 10^{4}$ ) were placed into the basolateral chamber containing 15\% FBS-DMEM while HCT-116 cells $\left(1 \times 10^{5}\right)$ in the root apical chamber with $10 \%$ FBS-DMEM. The medium was renewed every 1-2 days during the co-culture (4-5 days).

HCT-116 were co-cultured with MSCs which were transfected with NC-mimic, miR-34a-5p-mimic, si-NC, si-cMYC, miR-34a-5p-mimic + oe-NC, and miR-34a-5p-mimic + oe-c-MYC [33].

\section{3-(4, 5-dimethylthiazol-2-yl)-2, 5-diphenyltetrazolium bromide (MTT) assay}

HCT-116 cells were seeded into 96-well plates. The culture medium $(100 \mu \mathrm{L})$ was replaced with an equal volume of fresh medium containing $0.5 \mathrm{mg} / \mathrm{mL}$ MTT. After incubation of 0 , 24,48 , and $72 \mathrm{~h}, 100 \mu \mathrm{L}$ dimethyl sulfoxide (Sigma-Aldrich) was supplemented into cells and optical density $570 \mathrm{~nm}$ was measured by a microplate reader (Bio-Rad, Hercules, CA, USA) [27].

\section{Flow Cytometry}

Apoptosis HCT-116 cells were suspended in an Annexin Vfluorescein isothiocyanate (FITC) binding buffer, stained with Annexin V-FITC (Annexin V-FITC; Solarbio) and PI (Solarbio), and analyzed by a flow cytometer (BD Biosciences) [34].

\section{Transwell assay}

Cell migration and invasion abilities were evaluated by transwell assay. Cells were suspended in serum-free medium and added into the upper cavity with diluted matrix gel $(8-\mu \mathrm{m}$, BD Biosciences). The lower cavity contained $20 \%$ FBS. Cells after 48 -h incubation were fixed with $4 \%$ paraformaldehyde, stained with $0.1 \%$ crystal violet and counted in five randomlyselected fields [35].

\section{Reverse Transcription Quantitative Polymerase Chain Reaction}

Trizol reagent (Invitrogen) was employed to isolate total RNA from cells and tissues. mRNA and miRNA were reverse-transcribed into cDNA using the PrimerScript ${ }^{\mathrm{TM}}$ RT Master Mix Kit and the PrimerScript miRNA RT-PCR kit and treated with qPCR with SYBR Green method on the CFX96 real-time PCR system (Bio-Rad). Glyceraldehyde-3-phosphate dehydrogenase (GAPDH) and U6 were internal controls. Table 2 listed all the primers. Data were collected through ABI 7500 real-time PCR machine (ABI, Foster City, CA, USA) and analyzed by the $2^{-\Delta \Delta \mathrm{Ct}}$ method [36, 37].

\section{Western Blot Assay}

Total protein extracted from cells or tissues was lysed, separated by $6 \%$ sodium dodecyl sulfate-polyacrylamide gel electrophoresis, transferred to a polyvinylidene fluoride membrane, and blocked with 5\% skimmed milk. The primary antibodies including c-MYC (1:1000, sc-40, Santa Cruz Biotechnology), DNMT3a (1:2000, NB120-13888, Novus Biologicals), PTEN (1:1000, 9559, Cell Signaling Technology), CD9 (1:1000, ab92726), CD63 (1:1000, ab59479), Calnexin (1:1000, ab22595, Abcam), HSP70 (1:1000, 4872, Cell Signaling Technology), and $\beta$-actin (1:1000, sc-47778, Santa Cruz Biotechnology), along with secondary antibody (7074, 1:2000, Cell Signaling Technology) were applied to incubate with the membrane. The intensity of the band was quantified by Image analysis system (Quantity One v4.62, Bio-Rad) [38]. 
Table 2 Primer sequences

\begin{tabular}{llll}
\hline Genes & Forward $\left(5^{\prime}-3^{\prime}\right)$ & Tm & Reverse $\left(5^{\prime}-3^{\prime}\right)$ \\
\hline GAPDH & CATCCATGACAACTTTGGTATCGT & 60.8 & CATGAGGTCCAC \\
miR-34a-5p & TGGCAGTGTCTTAGCTGGTTGT & 59.9 & Uni-miR qPCR Primer, included in the kit \\
DNMT3a & GCCCATTCGATCTGGTGATT & 59.5 & GGCGGTAGAACTCAAAGAAGAG \\
PTEN & ACACGACGGGAAGACAAGTT & 56.8 & TCCTCTGGTCCTGGTATGAAG \\
c-MYC & TCCGTCCTCGGATTCTCTGCTCT & 66.3 & GCCTCCAGCAGAAGGTGATCCA \\
E-cadherin & CAGCATCACTGGCCAAGGAGCTGA & 70.4 & GACCACACTGATGACTCCTGTGTCC \\
Vimentin & CCGACACTCCT ACAAGATTTAGA & 56.1 & CAAAGATTTATTGAAGCAGAACC \\
N-cadherin & TTTGATGGAGGTCTCCTAACACC & 60.0 & ACGTTTAACACGTTGGAAATGTG \\
Snail & CCTCAAGATGCACATCCGAAGCCA & 70.1 & AGGAGAAGGGCTTCTCGCCAGTGT \\
U6 & CTCGCTTCGGCAGCACA & 59.3 & AACGCTTCACGA \\
\hline
\end{tabular}

Note: GAPDH, glyceraldehyde-3-phosphate dehydrogenase; miR-34a-5p, microRNA-34a-5p; DNMT3a, DNA methyltransferase 3a; PTEN, phosphatase and tensin homolog deleted on chromosome 10

\section{Dual Luciferase Reporter Gene Assay}

The fragment of c-MYC 3'-UTR wild-type (WT) and mutant (MUT) containing miR-34a-5p-binding sites was cloned into pmirGLO (Promega, Madison, USA). HCT-116 cells were transfected with WT/MUT-c-MYC vector and miR-34a-5pmimic/NC-mimic through Lipofectamine 3000 (Life Technologies Corporation, Carlsbad, CA, USA). Promega dual luciferase system (Glomax 20/20, ATCC) was utilized to measure firefly and Renilla luciferase activities, thus to determine relative luciferase activity [39].

\section{Co-immunoprecipitation Assay}

Cell extracts were reacted with $\operatorname{IgG}$ and protein $\mathrm{A} / \mathrm{G}$ agarose to eliminate non-specific binding, added with $2 \mu \mathrm{g}$ DNMT3a antibody (1:2000, NB120-13888, Novus Biologicals) and centrifuged. Then, $\mathrm{A} / \mathrm{G}$ agarose were suspended in $2 \times$ sodium dodecyl sulfate and heated at $100^{\circ} \mathrm{C}$. The immunoprecipitated protein was analyzed by $12 \%$ sodium dodecyl sulfate polyacrylamide gel electrophoresis, transferred to a polyvinylidene fluoride membrane, and blocked overnight. c-MYC (1:1000, sc-40, Santa Cruz Biotechnology) and PTEN (1:50, 9559, Cell Signaling Technology) were diluted and reacted with the membrane, followed by incubation with the secondary antibody. Finally, data analysis was performed by Odyssey infrared imaging system (LiCorBioSciences, Lincoln, NE, USA) [40].

\section{Tumor Xenografts in Nude Mice}

Male BALB/c nude mice (4-5 weeks) were raised in an environment of specific pathogen-free grade. Mice were subcutaneously injected with HCT-116 cells $\left(1 \times 10^{6}\right)$ co-cultured with transfected MSCs in the right abdomen. Mice for control were injected with PBS-treated HCT-116 cells. The average tumor diameter was recorded regularly every 4 days. After 24 days, the mice were euthanized and the tumor weight was recorded [41-43].

\section{Statistical Analysis}

Statistical analysis was finished by SPSS 18.0 (SPSS, Chicago, USA). Analysis of variance was utilized to evaluate the statistical differences of groups. $P<0.05$ was considered a significant difference [44].

\section{Results}

\section{Identification of MSC and EV}

MSC-derived exosomes serve as the inhibitor in pancreatic cancer [33]. MSC obtained from patients undergoing hip arthroplasty showed plastic adhesion, growing like fibroblast-like spindles after 2-3 passages (Fig. 1A).

Viewed by a TEM, the extracted EV from MSC were about 50-130 nm (Fig. 1B). Moreover, Western blot identified the surface antigens of EV, and discovered that, CD9, CD63, and HSP70 were positively expressed while Calnexin was negatively expressed (Fig. 1C).

\section{miR-34a-5p is Downregulated in CRC}

It has been reported that miR-34a-5p is downregulated in CRC [15] while c-MYC is involved in the regulation of CC, showing a high expression trend [45]. miRNA can affect the tumor microenvironment by affecting the level of protein. DNMT3a can regulate the development of $\mathrm{CC}$, and generally shows a high expression trend in $\mathrm{CC}$ cells, and the regulation 
A

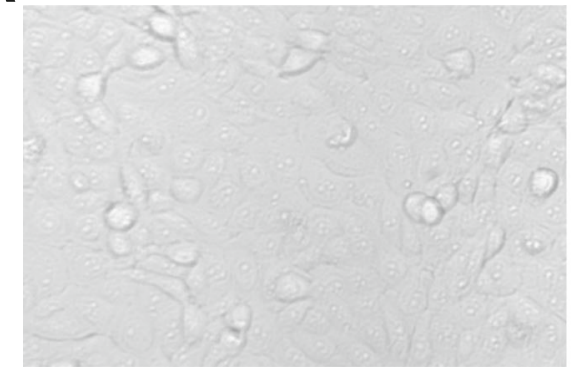

B

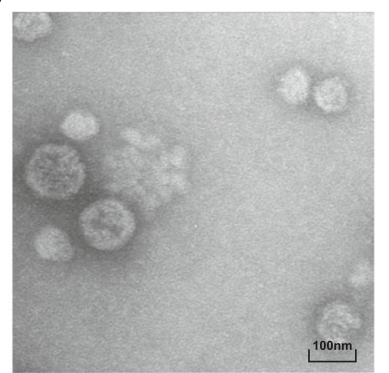

C

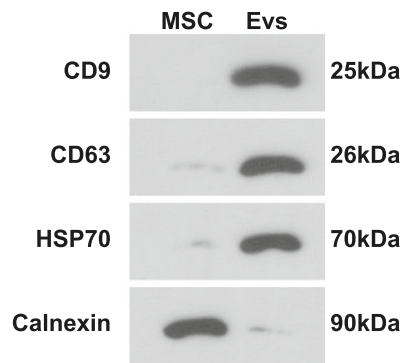

Fig. 1 Identification of MSC and EV. A Observation of MSCs; B TEM observed MSC-EV; C Western blot tested surface antigens of MSC and EV (CD9, CD63, HSP70, and Calnexin)

of DNMT3a may be related to the expression of miRNA [46]. At the same time, PTEN is lowly expressed in CC and is closely related to the development of CC [47]. RT-qPCR and Western blot tested the downregulated miR-34a-5p and PTEN, and upregulated c-MYC and DNMT3a in cancer tissues and cells (Fig. 2A-J). Among CRC cell lines, miR-34a$5 p$ and PTEN showed the lowest expression while c-MYC and DNMT3a showed the highest levels in HCT-116 cells, so HCT-116 cells were adopted for in vitro experiments.

\section{Inhibiting miR-34a-5p Promotes CRC Cell Development}

The effect of miR-34a-5p on the occurrence and development of CRC were clarified by various experiments. After successful transfection with miR-34a-5p inhibitor (Fig. 3A), HCT116 cell proliferation, migration and invasion were enhanced, and apoptosis was inhibited (Fig. 3B-E).

EMT contributes to the metastasis of cancer cells and is associated with more aggressive cancer phenotypes. EMTrelated proteins in $\mathrm{CC}$ are related to tumor development [48]. EMT is characterized by reduced level of epithelial marker E-cadherin, accompanied by increased expression of mesenchymal markers Vimentin, N-cadherin, and Snail [49]. As measured, E-cadherin level was decreased while Ncadherin, Vimentin, and Snail levels were raised in HCT116 cells after downregulating miR-34a-5p (Fig. 3F-I).

\section{MSC-EV Suppress CRC Progression}

MSC-EV mediate cancer development by changing tumor microenvironment [43]. Proved by PKH26 staining, HCT116 cells having been co-cultured with MSC-EV smoothly internalized MSC-EV (Fig. 4A). Based on the theory that MSC-EV can transfer miRNAs to other cells [50], HCT-116 cells were co-cultivated with MSC-EV that had been transfected with miR-34a-5p-mimic. Then, the results displayed that miR-34a-5p expression was increased in HCT-116 cells in a time-dependent manner (Fig. 4B).
After co-culture with MSC-EV, HCT-116 cells were characterized by inhibited cell viability, migration and invasion, promoted apoptosis, augmented E-cadherin expression, and reduced Vimentin, N-cadherin, and Snail expression (Fig. $4 \mathrm{C}-\mathrm{J})$.

\section{Inhibiting C-MYC Hinders CRC Cell Development}

Our experiments in tissues and cells further confirmed upregulation trend of c-MYC in CRC. To delve the mechanism of cMYC in CRC, si-c-MYC was successfully transfected into HCT-116 cells (Fig. 5A), after which HCT-116 cell progression and EMT were found to be retarded (Fig. 5B-I).

\section{MSC-EV Carrying Upregulated miR-34a-5p or Downregulated c-MYC Further Disrupt CRC Cell Progression}

EV from MSC transfected with miR-34a-5p mimic or si-cMYC were co-cultured with CRC cells for further research. It was confirmed that MSC-EV-containing miR-34a-5p mimic or si-c-MYC further limited CRC cell growth and EMT process (Fig. 6A-H). The results informed that MSC-EV carrying upregulated miR-34a-5p or downregulated c-MYC further disrupted CRC cell progression.

\section{miR-34a-5p Targets c-MYC}

Though the regulatory functions of miR-34a-5p and c-MYC have been clarified in CRC, their combined action is still unknown. Given that, we surveyed whether c-MYC is involved in miR-34a-5p regulating CRC. Firstly, c-MYC level after transfection of miR-34a-5p inhibitor was measured and the finding manifested the incremental c-MYC level in cells (Fig. 7A). Later, the targeting relationship of miR-34a-5p and c-MYC was predicted on the Starbase website (Fig. 7B) and further confirmed by dual luciferase report experiment (Fig. 7C). 

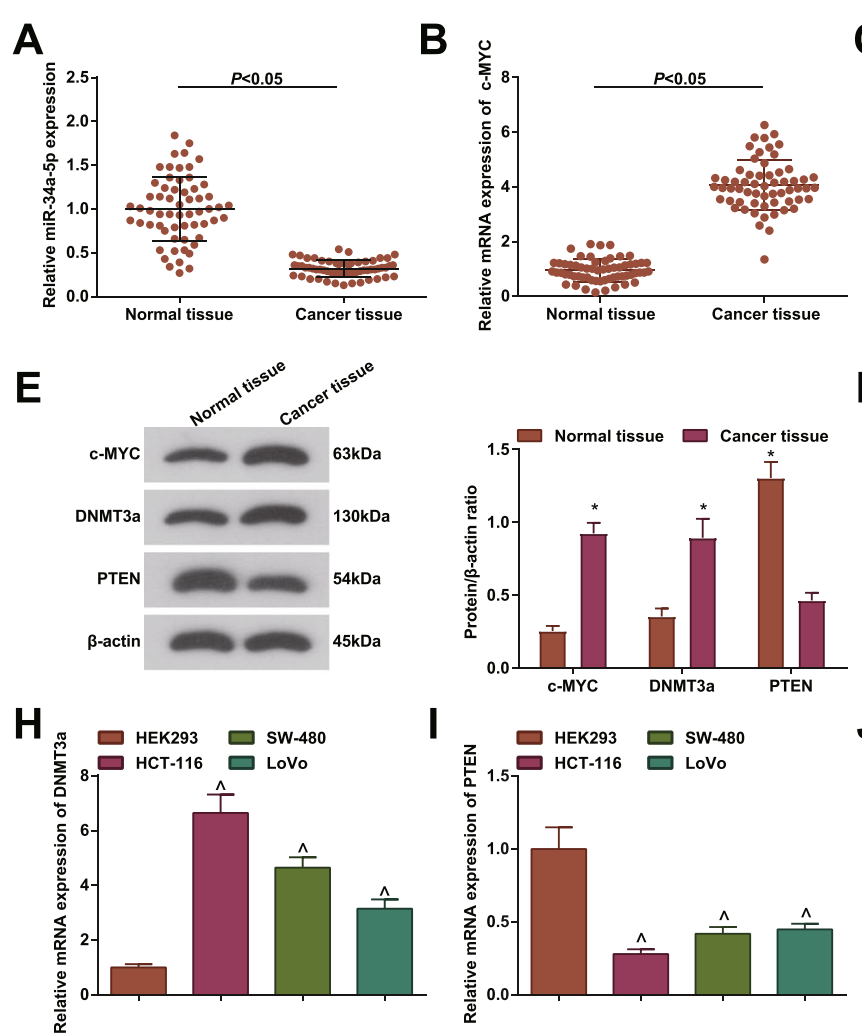

Fig. 2 miR-34a-5p is down-regulated in CRC. A-D RT-qPCR tested miR-34a-5p, c-MYC, DNMT3a and PTEN mRNA expression in cancer tissues and normal tissues, cases $=60 ; \mathbf{E}$ Western blot tested $\mathrm{c}-$ MYC, DNMT3a, and PTEN protein expression in cancer tissues and normal tissues; F-I. RT-qPCR tested miR-34a-5p, c-MYC, DNMT3a, and PTEN mRNA expression in HEK293, HCT-116, SW-480, and LoVo

There is an interaction between c-MYC and DNMT3a [21]. In our study, their interaction was also validated. DNMT3a expression was reduced after inhibiting c-MYC (Fig. 7D). Moreover, two-way COIP assay mirrored that c-MYC and DNMT3a were coprecipitated in HCT-116 cells by anti-c-MYC antibody. Co-immunoprecipitation with anti-DNMT3a antibody further confirmed their interaction (Fig. 7E). PTEN has been mentioned as the mediated gene of DNMT3a [24]. Actually, CO-IP assay also validated their interaction (Fig. 7F).

\section{c-MYC Overexpression Abrogates EV-containing miR- 34a-5p Upregulation-induced Effects on CRC}

The combined effects of MSC-EV, miR-34a-5p, and c-MYC were further discussed in the field of CRC. Series of assays experimentally presented that though MSC-EV delivering miR-34a-5p-mimic depressed CRC cell growth and EMT process, another transfection of oe-c-MYC into MSC-EV would abrogate the therapeutic effects of miR-34a-5p-mimic (Fig. $8 \mathrm{~A}-\mathrm{H})$.
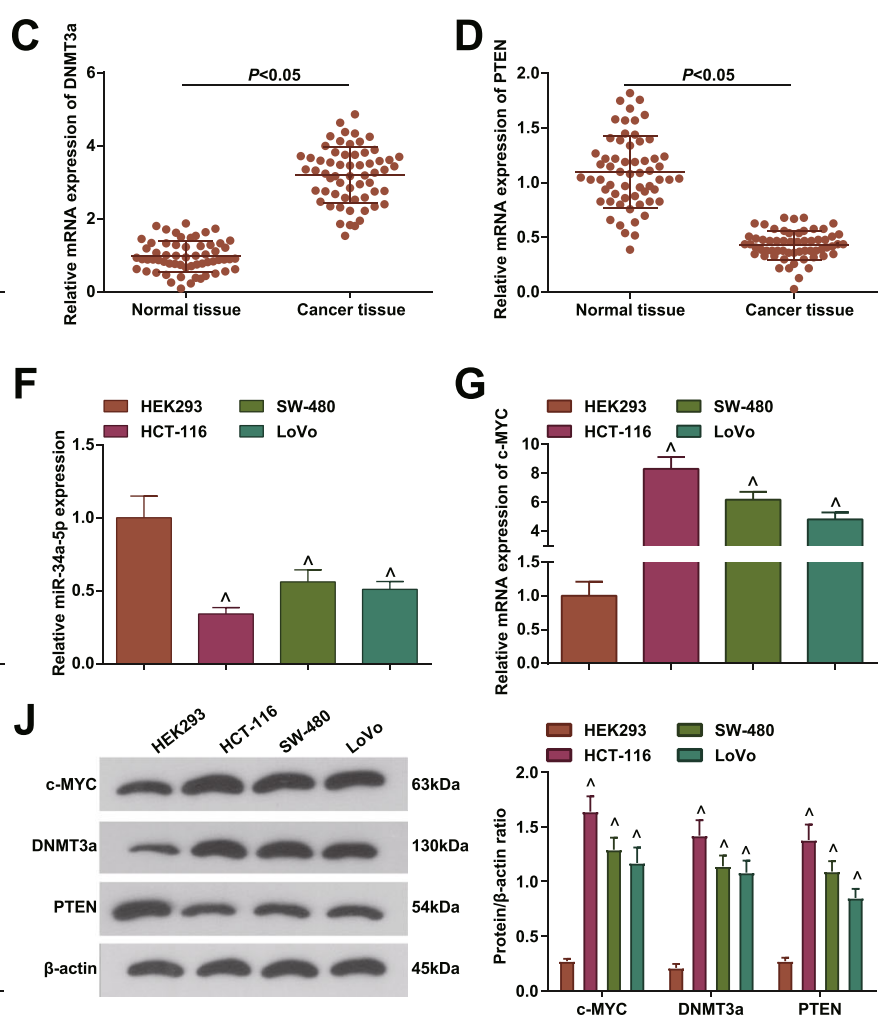

cells; J Western blot tested c-MYC, DNMT3a and PTEN protein expression in HEK293, HCT-116, SW-480 and LoVo cells; The data were expressed as mean \pm standard deviation of three independent experiments. ${ }^{*} P<0.05$ compared with normal tissues; ${ }^{\wedge} P<0.05$ compared with HEK293 cells

\section{Restoring miR-34a-5p or Depleting c-MYC in MSC-EV Limits Tumor Formation in CRC}

Tumor xenograft in nude mice was carried out to study whether miR-34a-5p/c-MYC/DNMT3a axis regulates CRC in vivo. HCT-116 cells co-cultured with MSC-EV were injected into mice ( $n=5 /$ group). The results revealed that MSC-EV reduced tumor volume and weight in mice, and MSC-EV transporting miR-34a-5p-mimic further enhanced the tumorsuppression effects. In addition, the anti-tumor effect of MSCEV-derived miR-34a-5p was reversed by c-MYC overexpression (Fig. 9A, B).

Sections of tumors were analyzed by HE staining. It was displayed that the tumor tissues without any treatment were necrotic and accompanied by inflammatory cell infiltration, and the tumor cells were irregular in size and shape. The tumor tissues from mice injected with HCT-116 cells co-cultured with MSC$\mathrm{EV}$ showed attenuated inflammatory cell infiltration. In response to upregulation of miR-34a-5p or downregulation of c-MYC on the basis of MSC-EV treatment, the degree of inflammatory cell infiltration in tissue sections was the lowest (Fig. 9C). It was indicated that miR-34a-5p/c-MYC/DNMT3a axis regulated PTEN to suppress CRC tumor growth in vivo. 
A

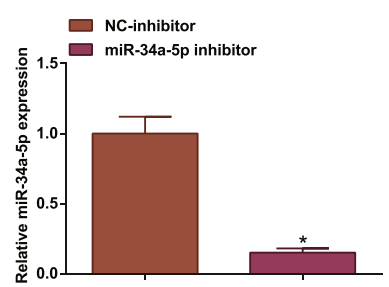

D

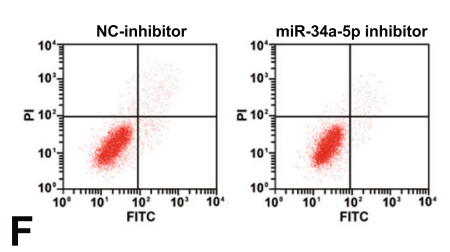

NC-inhibitor

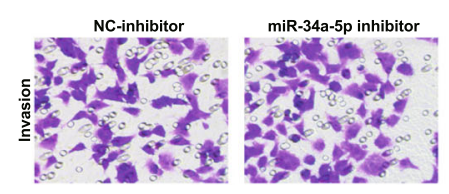

I

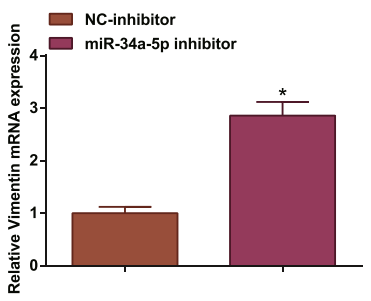

B
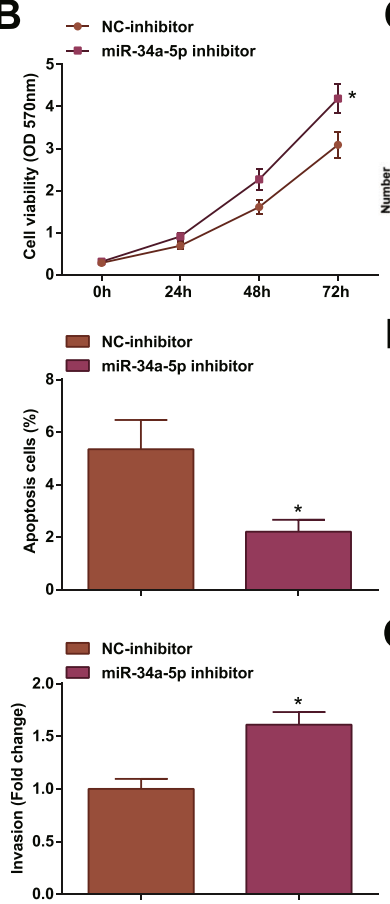

$\mathbf{J}$

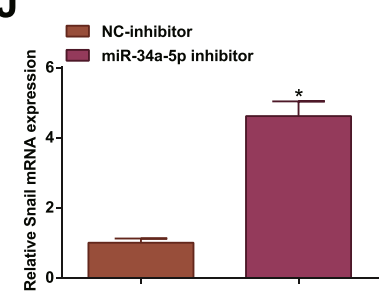

Fig. 3 Inhibiting miR-34a-5p promotes CRC cell development. A RTqPCR tested miR-34a-5p expression in HCT-116 cells after miR-34a-5p inhibition; B MTT tested viability of HCT-116 cells after miR-34a-5p inhibition; C flow cytometry tested apoptosis of HCT-116 cells after miR-34a-5p inhibition; D Transwell assay tested migration of HCT-116 cells after miR-34a-5p inhibition; $\mathbf{E}$ Transwell assay tested invasion of HCT-116 cells after miR-34a-5p inhibition; F RT-qPCR tested Ecadherin mRNA expression in HCT-116 cells after miR-34a-5p

\section{Discussion}

CRC is the $3^{\text {rd }}$ prevalent malignancy, in which metastasis is the main cause of cancer-related deaths [51]. Our study has delineated the action of miR-34a-5p/c-MYC/ DNMT3a/PTEN axis in CRC. At the beginning, we made efforts to discuss the role of miR-34a-5p in $\mathrm{CRC}$ and then discovered that miR-34a-5p was downregulated in the disease and its downregulation promoted on cellular growth and EMT process. Next, we confirmed $c-M Y C$ as the target of miR-34a-5p and knocking down c-MYC repressed CRC development and EMT. Subsequently, we also defined MSC-EV as a protector against $\mathrm{CRC}$, while the combined function of MSC-EV with upregulated miR-34a-5p or

C
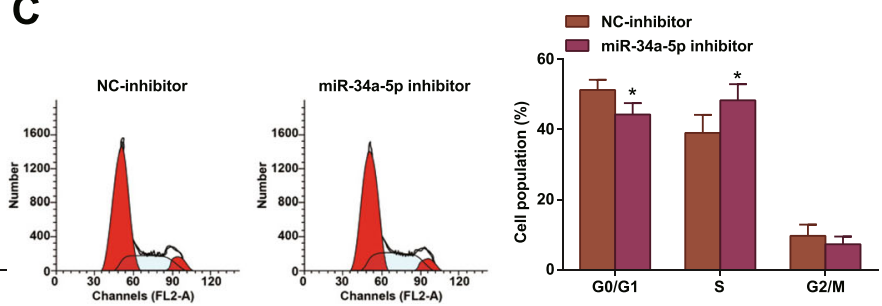

E
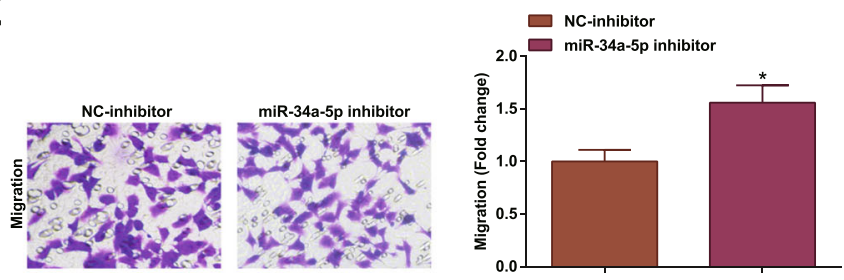

G

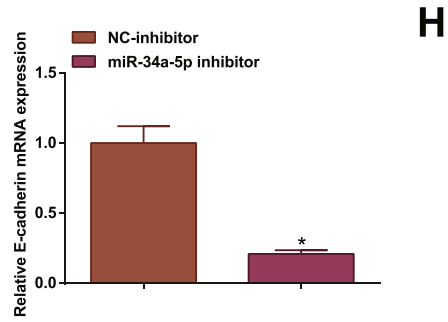

H

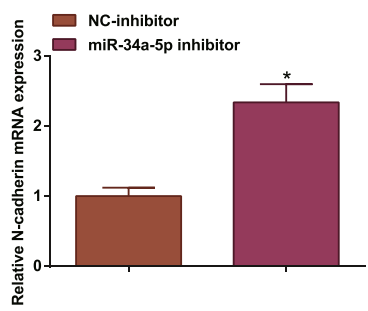

inhibition; G RT-qPCR tested N-cadherin mRNA expression in HCT116 cells after miR-34a-5p inhibition; H RT-qPCR tested Vimentin mRNA expression in HCT-116 cells after miR-34a-5p inhibition; I RTqPCR tested Snail mRNA expression in HCT-116 cells after miR-34a-5p inhibition; the data were expressed as mean \pm standard deviation of three independent experiments. ${ }^{*} P<0.05$ compared with the $\mathrm{NC}$-inhibitor group

downregulated c-MYC was better to control CRC in vitro and in vivo (Supplementary Figure S1).

miR-34a-5p has been marked with anti-tumor effects in cancers, including but not limited to CRC. In a latest report, suppressed miR-34a-5p showcases in CRC and long non-coding RNA-mediated miR-34a-5p downregulation is attributable to strengthen invasion and metastasis of CRC cells [52]. A preceding paper has mentioned the reduced level of miR-34a-5p in CRC, and increased miR-34a-5p level introduced to CRC cells would limit cell proliferation, migration, invasion, and metastasis, as well as induce cell apoptosis [15]. In the area of prostate cancer, miR-34a-5p expression is lower than the basic level and lncRNA-meidated knockdown of miR$34 a-5 p$ can elevate disheveled associated activator of 

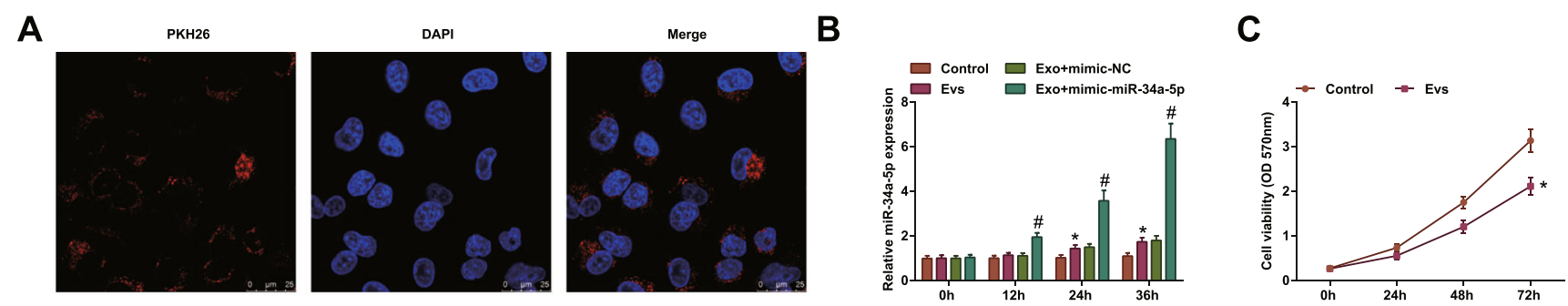
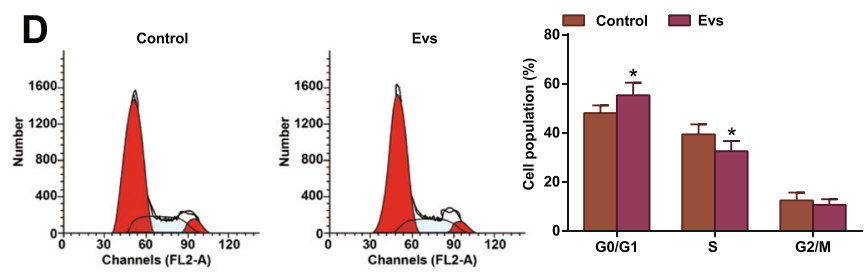

$\mathbf{F}$
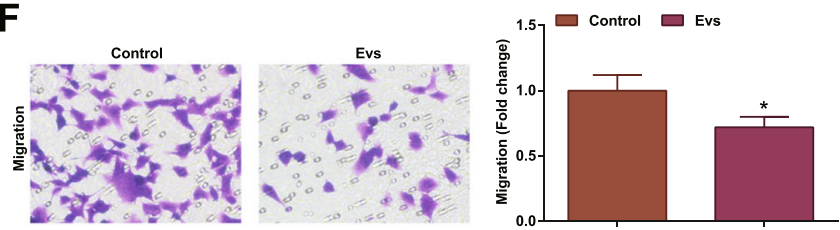

$H$
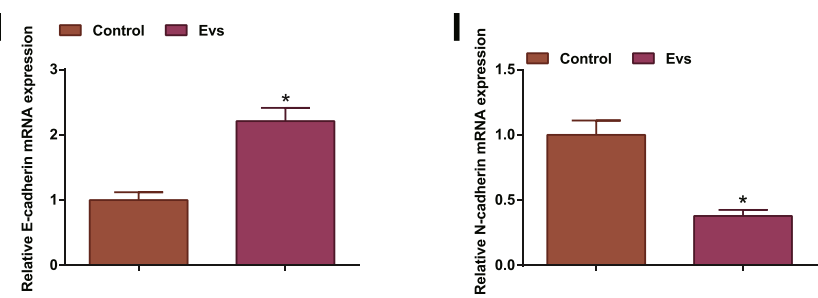

Fig. 4 MSC-EV suppress CRC progression. A PKH26 staining observed uptake of EV; B RT-qPCR tested miR-34a-5p expression in HCT-116 cells after co-culture of EV; C MTT tested viability of HCT-116 cells after co-culture of EV; D flow cytometry tested apoptosis of HCT-116 cells after co-culture of EV; $\mathbf{E}$ Transwell assay tested migration of HCT116 cells after co-culture of EV; $\mathbf{F}$ Transwell assay tested invasion of HCT-116 cells after co-culture of EV; G RT-qPCR tested E-cadherin
E
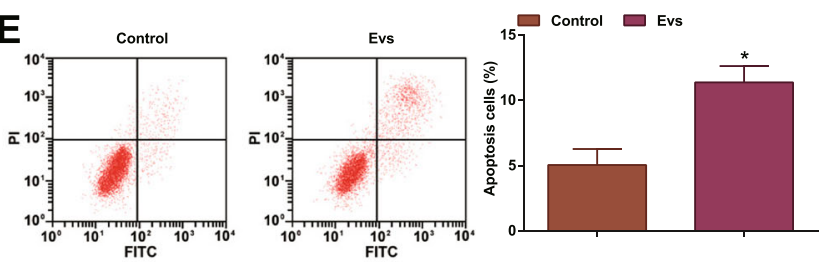

G
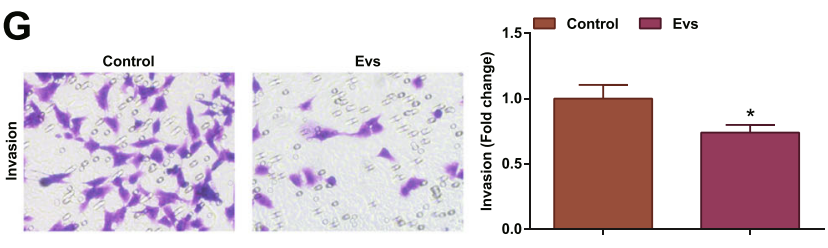

J
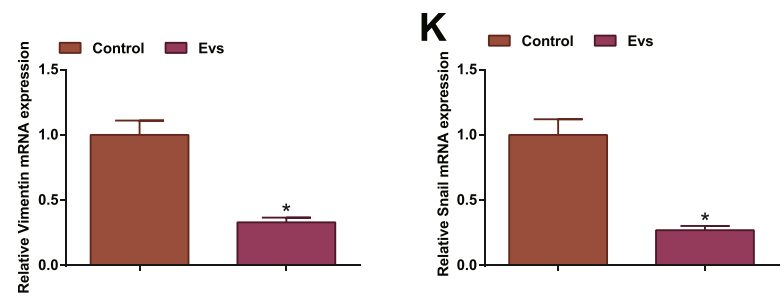

mRNA expression in HCT-116 cells after co-culture of EV; H RTqPCR tested N-cadherin mRNA expression in HCT-116 cells after coculture of EV; I RT-qPCR tested Vimentin mRNA expression in HCT116 cells after co-culture of EV; J RT-qPCR tested Snail mRNA expression in HCT-116 cells after co-culture of EV; the data were expressed as mean \pm standard deviation of three independent experiments. ${ }^{*} P<0.05$ compared with the control group morphogenesis 1 , thus to accelerate cancer development [53]. As to liver cancer, miR-34a-5p exerts anti-proliferative, anti-migratory, and anti-invasive effects on cancer cells through targeted regulation of Yin and Yang 1 [54]. As for esophageal squamous cell carcinoma, cancer cells containing upregulated miR-34a-5p exhibit the impaired proliferation, migration, invasion, and EMT [55]. As to the combined functions of miRNA with EV, a published article has unraveled that miR-34a can be delivered by human bone marrow MSC-derived exosomes into glioblastoma cells, thereby disrupting cell proliferation, invasion, migration, and tumorigenesis [56]. To our surprise, MSC-derived exosomal miR-34c into nasopharyngeal carcinoma exerts to frustrate cell aggressiveness and EMT process [57]. Other than that, MSC-derived exosomes could convey miR-3940-5p overexpression vector to CRC cells, thus to obstruct EMT process, cell invasion, and tumor metastasis [58]. Besides, bone marrow MSC-derived exosomes functionally cooperate with miR-124 in pancreatic adenocarcinoma cells, so as to inhibit cell proliferation and EMT [59]. All of the reports support the positive role of miR-34a-5p in cancer prevention, as well as the collaborative effects of EV and miRNAs on cancers.

It has been fundamentally based that miR-34 can target cMyc $[60,61]$. In fact, similar to our finding in CRC, another report studying high-grade colon adenocarcinoma cells has examined the widespread c-MYC [62]. Pivoted on the actual role in cancer development, a study has once recorded that induction of c-Myc expression facilitates RING finger protein 8 to promote CC cell proliferation [63]. Oppositely, an inhibitor for c-MYC, compound 42 has been developed to 
A

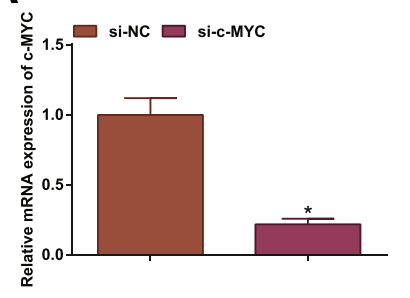

D
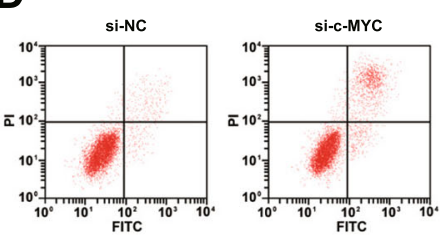

$\mathbf{F}$
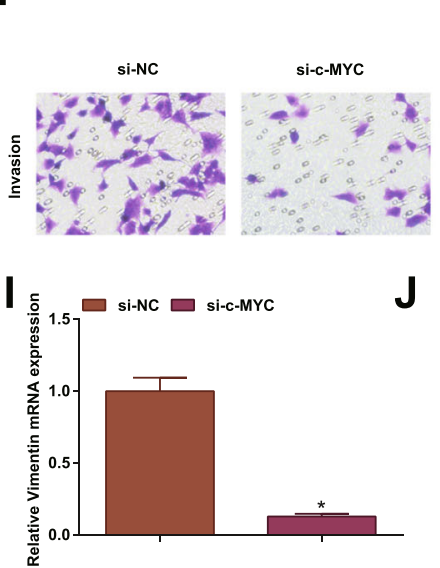

J

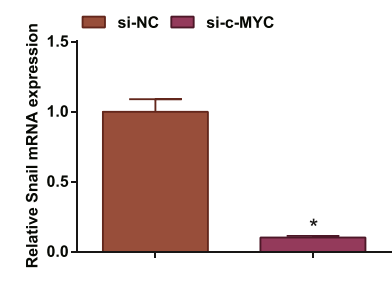

Fig. 5 Inhibiting c-MYC hinders CRC cell development. A RT-qPCR tested c-MYC expression in HCT-116 cells after c-MYC inhibition; B MTT tested viability of HCT-116 cells after c-MYC inhibition; $\mathbf{C}$ flow cytometry tested apoptosis of HCT-116 cells after c-MYC inhibition; D Transwell assay tested migration of HCT-116 cells after c-MYC inhibition; E Transwell assay tested invasion of HCT-116 cells after cMYC inhibition; F RT-qPCR tested E-cadherin mRNA expression in
C
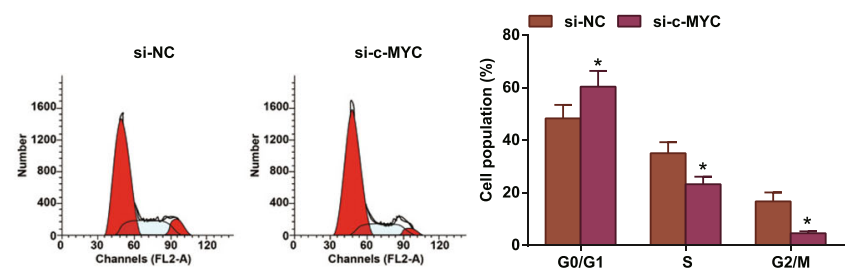

E

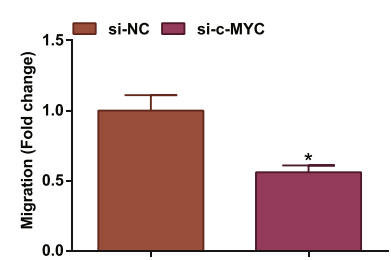

G

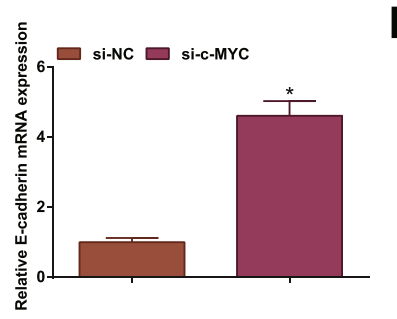

$\mathbf{H}$

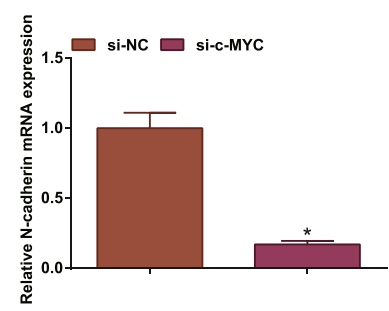

HCT-116 cells after c-MYC inhibition; G RT-qPCR tested N-cadherin mRNA expression in HCT-116 cells after c-MYC inhibition; H RTqPCR tested Vimentin mRNA expression in HCT-116 cells after cMYC inhibition; I RT-qPCR tested Snail mRNA expression in HCT116 cells after c-MYC inhibition; the data were expressed as mean \pm standard deviation of three independent experiments. ${ }^{*} P<0.05$ compared with the si-NC group

report has decoded that MYC interacts with DNMT3a, thus silencing miR-200b and leading to EMT in triplenegative breast cancer [67]. DNMT3a is defined as one of the overexpressed genes in sporadic CRC [22] and theaflavin, an inhibitor for DNMT (DNMT1 and DNMT3a) can combat CC cell proliferation and progression [68]. It is interestingly identified that DNMT3a upregulation partially reduces PTEN level in hepatocellular carcinoma cells [69], showing the inverse relation between DNMT3a and PTEN. PTEN is an actor that strains CRC cell proliferation and migration [70] and PTEN upregulation could prevent proliferation and induce apoptosis of chemo-resistant CC cells [71]. All in all, the potential performances of DNMT3a and PTEN have been further studied in CRC. 


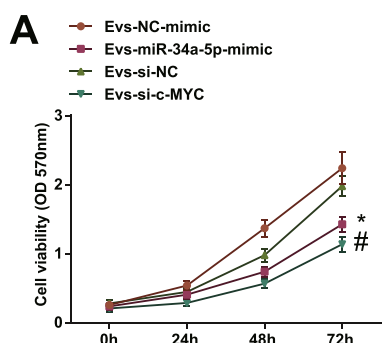

B
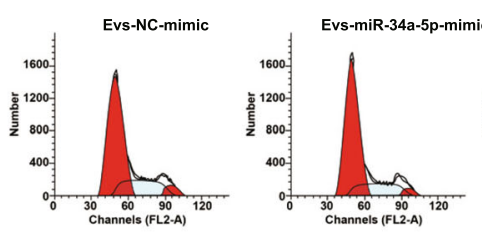

C
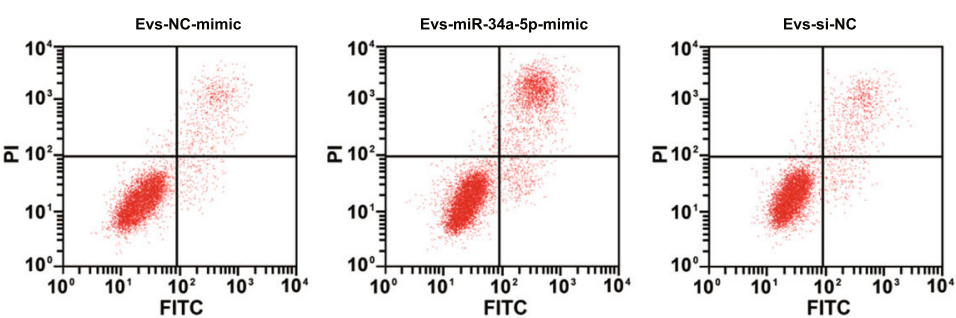

D
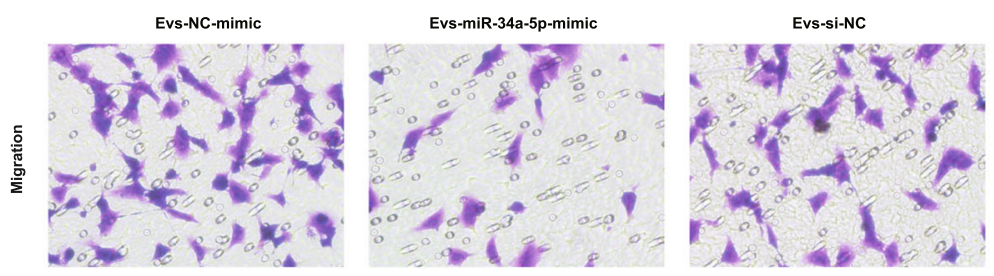

$\mathbf{E}$
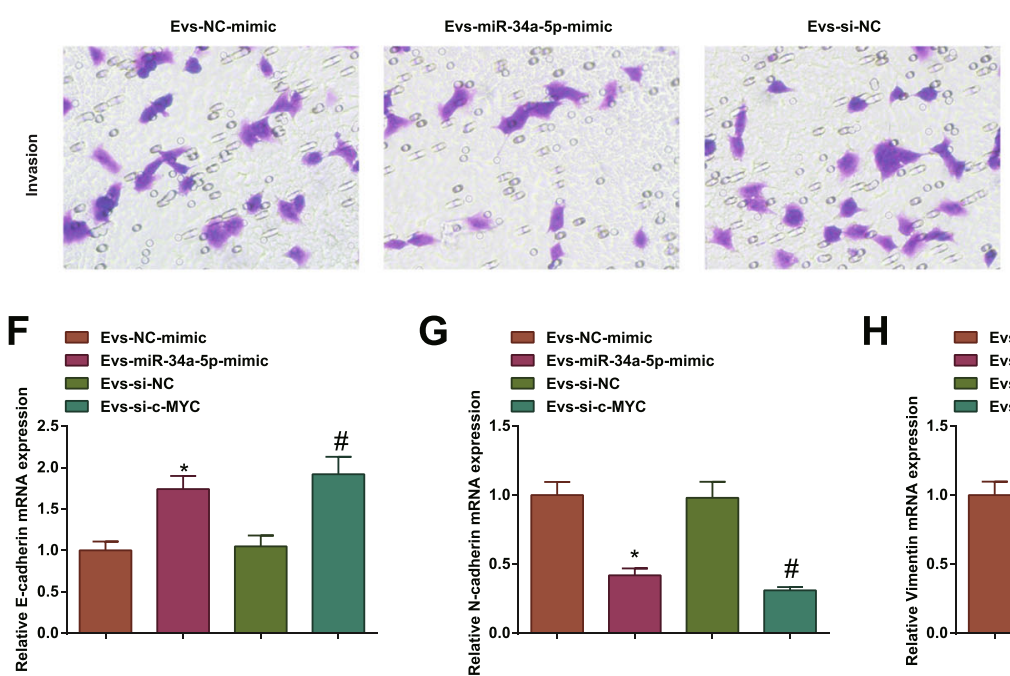

Fig. 6 MSC-EV carrying upregulated miR-34a-5p or downregulated cMYC further disrupt CRC cell progression. A MTT tested viability of HCT-116 cells after co-culture of EV; $\mathbf{B}$ flow cytometry tested apoptosis of HCT-116 cells after co-culture of EV; C Transwell assay tested migration of HCT-116 cells after co-culture of EV; D Transwell assay tested invasion of HCT-116 cells after co-culture of EV; E RT-qPCR tested E-cadherin mRNA expression in HCT-116 cells after co-culture

\section{Conclusion}

To conclude, miR-34a-5p is anti-tumor in CRC through targeting c-MYC to control DNMT3 and PTEN. It is a
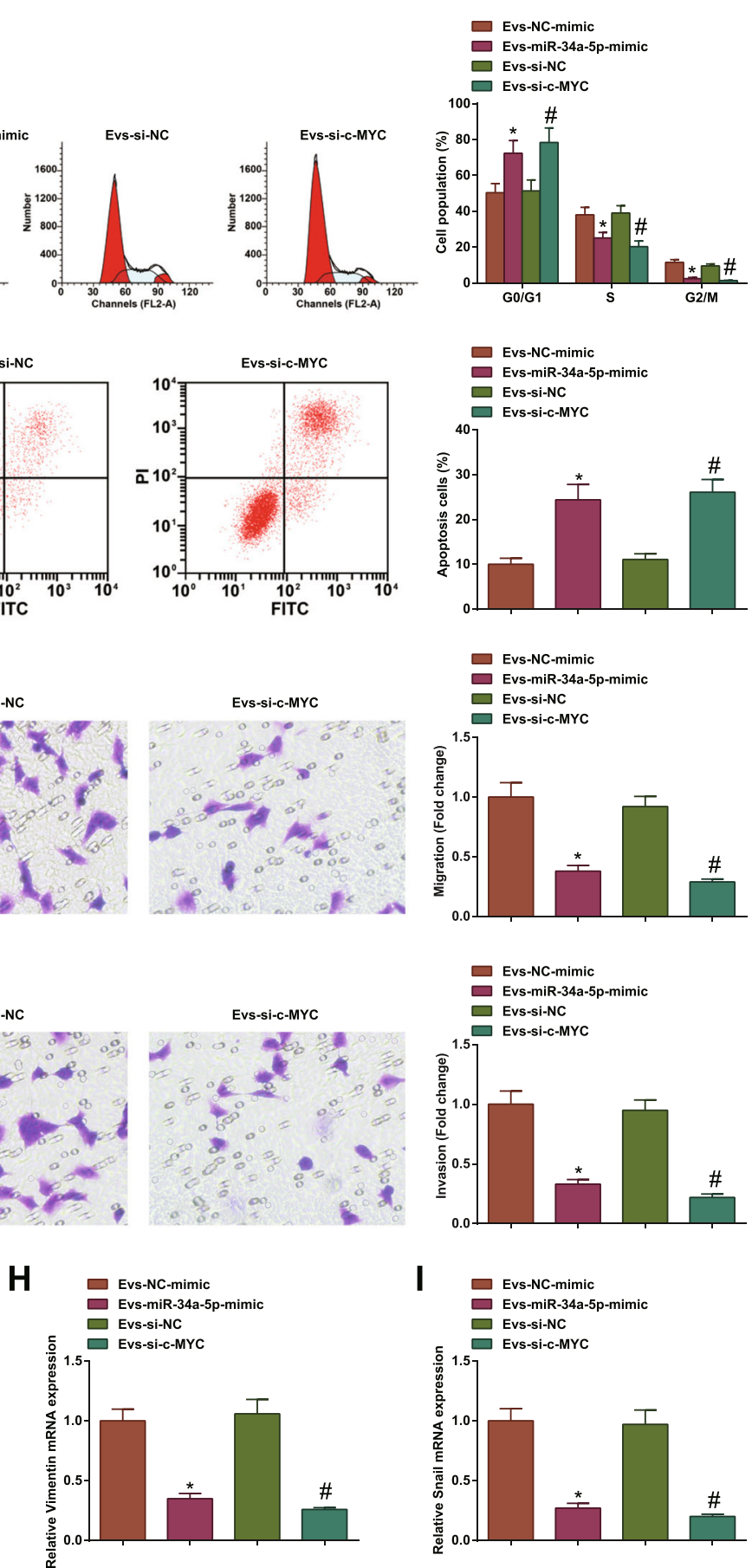

of EV; F RT-qPCR tested N-cadherin mRNA expression in HCT-116 cells after co-culture of EV; G RT-qPCR tested Vimentin mRNA expression in HCT-116 cells after co-culture of EV; H RT-qPCR tested Snail mRNA expression in HCT-116 cells after co-culture of EV; the data were expressed as mean \pm standard deviation of three independent experiments. ${ }^{*} P<0.05$ compared with the EV-NC-mimic group; ${ }^{\#} P<$ 0.05 compared with the EV-si-NC group

novel research to navigate the mechanisms underlying CRC pathology, and more experimental researches are still needed for better understanding the miR-34a-5prelated mechanism in cancers. 


\section{A}

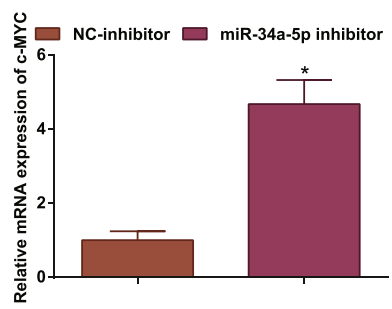

$\mathbf{D}^{\mathrm{g}}$

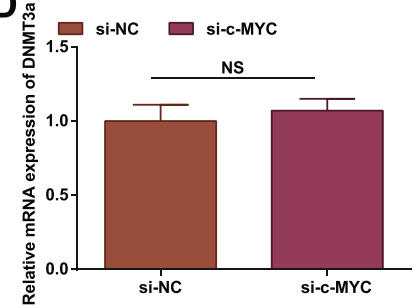

B

Target: $\quad$ ' uUAGCCA--UAAUGUAAACUGCCu 3'

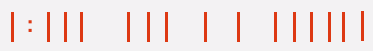

miRNA : 3' ugUUGGUCGAUU-C-UGUGACGGu $5^{\prime}$
C

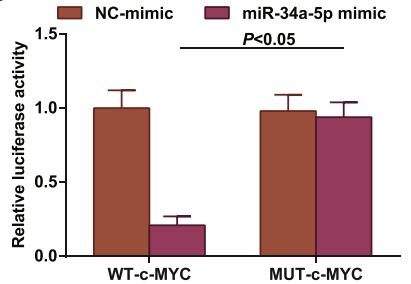

$\mathbf{E}$

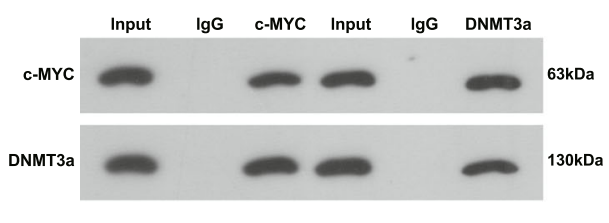

$\mathbf{F}$

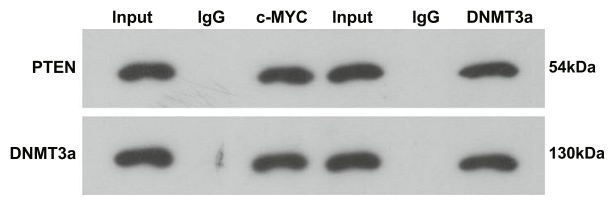

Fig. 7 miR-34a-5p targets c-MYC. A RT-qPCR tested c-MYC expression in HCT-116 cells after miR-34a-5p inhibition; B Bioinformatics website Starbase predicted the binding site of miR-34a$5 \mathrm{p}$ and $\mathrm{c}-\mathrm{MYC} ; \mathbf{C}$ luciferase reporter gene experiment verified the targeting relationship between miR-34a-5p and c-MYC; D RT-qPCR tested DNMT3a mRNA expression in HCT-116 cells after c-MYC inhibition; E CO-IP assay tested the interaction between c-MYC and DNMT3a; F CO-IP assay tested the interaction between PTEN and DNMT3a; the data were expressed as mean \pm standard deviation of three independent experiments. ${ }^{*} P<0.05$ compared with the NCinhibitor group
A

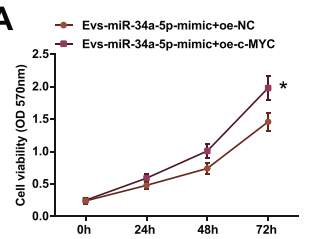

D

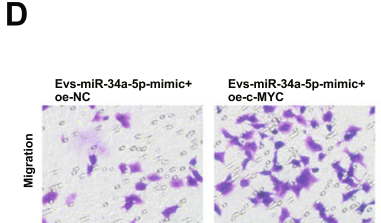

$\mathbf{G}$

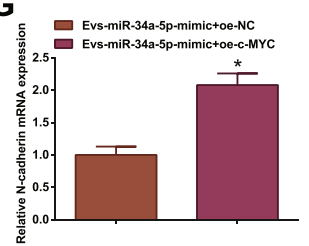

H
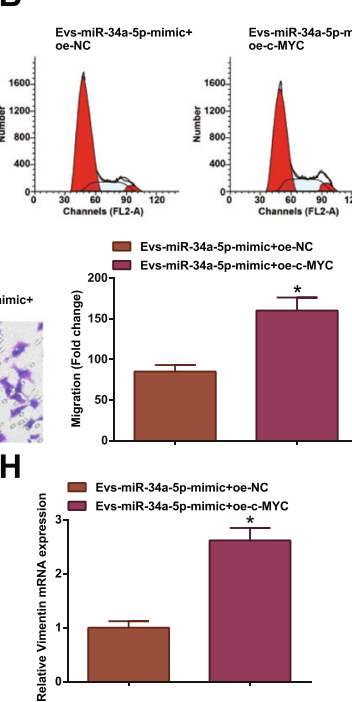

Fig. 8 c-MYC overexpression abrogates EV-mediated miR-34a-5p upregulation-induced effects on CRC. A MTT tested viability of HCT116 cells after co-culture of $\mathrm{EV}$; $\mathbf{B}$ flow cytometry tested apoptosis of HCT-116 cells after co-culture of EV; $\mathbf{C}$ Transwell assay tested migration of HCT-116 cells after co-culture of EV; D Transwell assay tested invasion of HCT-116 cells after co-culture of EV; E RT-qPCR tested E-cadherin mRNA expression in HCT-116 cells after co-culture of EV;
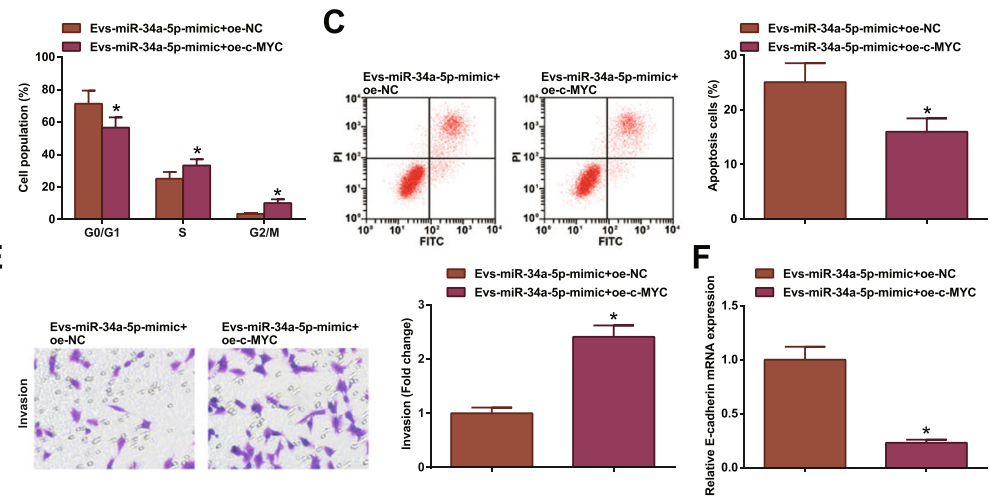

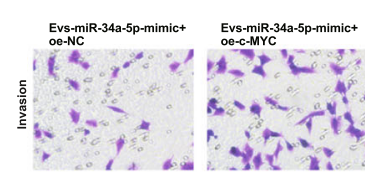

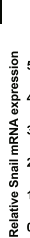

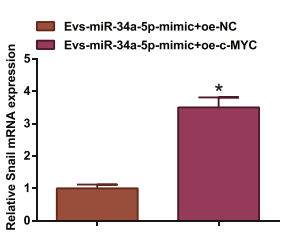

F RT-qPCR tested N-cadherin mRNA expression in HCT-116 cells after co-culture of EV; G RT-qPCR tested Vimentin mRNA expression in HCT-116 cells after co-culture of EV; H RT-qPCR tested Snail mRNA expression in HCT-116 cells after co-culture of EV; the data were expressed as mean \pm standard deviation of three independent experiments. ${ }^{*} P<0.05$ compared with the EV-miR-34a-5p-mimic + oe-NC group 


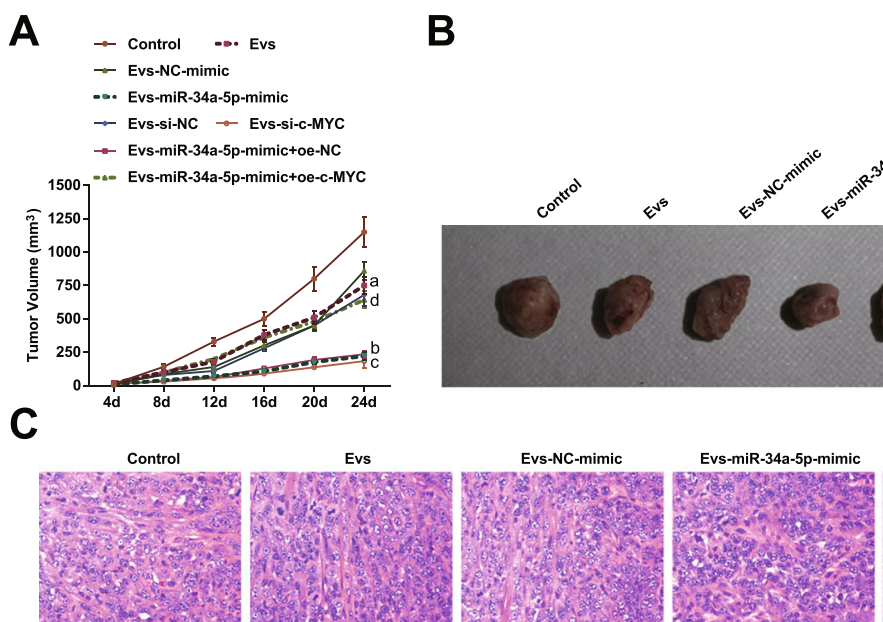

Fig. 9 Restoring miR-34a-5p or depleting c-MYC in MSC-EV limits tumor formation in CRC. A Tumor volume of nude mice; $\mathbf{B}$ tumors and tumor weight of nude mice; C HE staining detected the histopathology of tumors; the data were expressed as mean \pm standard deviation of three

Supplementary Information The online version contains supplementary material available at https://doi.org/10.1007/s12035-021-02431-9.

Authors' contributions Jiangning Zhao contributed to study design; Jiangning Zhao contributed to manuscript editing; Huanrong Lin contributed to experimental studies; Kunsong Huang contributed to data analysis.

\section{Declaration}

Ethics Approval and Consent to Participate The research protocol was approved by the ethics committee of The Fourth Clinical Medical School of Guangzhou University of Chinese Medicine; Shenzhen Traditional Chinese Medicine Hospital, and written informed consent was obtained. All animal experiments were conducted with the guidelines for the care and use of laboratory animals of the National Institutes of Health.

Conflict of Interest The authors declared no potential conflicts of interest with respect to the research, authorship, and publication of this article.

Open Access This article is licensed under a Creative Commons Attribution 4.0 International License, which permits use, sharing, adaptation, distribution and reproduction in any medium or format, as long as you give appropriate credit to the original author(s) and the source, provide a link to the Creative Commons licence, and indicate if changes were made. The images or other third party material in this article are included in the article's Creative Commons licence, unless indicated otherwise in a credit line to the material. If material is not included in the article's Creative Commons licence and your intended use is not permitted by statutory regulation or exceeds the permitted use, you will need to obtain permission directly from the copyright holder. To view a copy of this licence, visit http://creativecommons.org/licenses/by/4.0/.

\section{References}

1. Swierczynski M et al (1875) New insights into molecular pathways in colorectal cancer: adiponectin, interleukin-6 and opioid signaling. Biochim Biophys Acta Rev Cancer 2020(1):188460 independent experiments. a $P<0.05$ compared with the control group; b $P<0.05$ compared with the EV-NC-mimic group; c $P<0.05$ compared with the EV-si-NC group; $\mathrm{d} P<0.05$ compared with the EV-miR-34a-5pmimic + oe-NC group

2. Akimoto $\mathrm{N}$ et al (2020) Rising incidence of early-onset colorectal cancer - a call to action. Nat Rev Clin Oncol

3. Yu X, Zhu L, Liu J, Xie M, Chen J, Li J (2020) Emerging role of immunotherapy for colorectal cancer with liver metastasis. Onco Targets Ther 13:11645-11658

4. Cimino SK, Eng C (2020) Up-and-coming experimental drug options for metastatic colorectal cancer. J Exp Pharmacol 12:475485

5. Dalal N, Jalandra R, Sharma M, Prakash H, Makharia GK, Solanki PR, Singh R, Kumar A (2020) Omics technologies for improved diagnosis and treatment of colorectal cancer: Technical advancement and major perspectives. Biomed Pharmacother 131:110648

6. van Niel G, D'Angelo G, Raposo G (2018) Shedding light on the cell biology of extracellular vesicles. Nat Rev Mol Cell Biol 19(4): 213-228

7. Akbari A, Jabbari N, Sharifi R, Ahmadi M, Vahhabi A, Seyedzadeh SJ, Nawaz M, Szafert S et al (2020) Free and hydrogel encapsulated exosome-based therapies in regenerative medicine. Life Sci 249:117447

8. Xing H, Liang C, Xu X, Sun H, Ma X, Jiang Z (2020) Mesenchymal stroma/stem-like cells of GARP knockdown inhibits cell proliferation and invasion of mouse colon cancer cells (MC38) through exosomes. J Cell Mol Med 24:13984-13990

9. Ahmadi M, Rezaie J (2020) Tumor cells derived-exosomes as angiogenenic agents: possible therapeutic implications. J Transl Med 18(1):249

10. Ahmadi M, Jafari R, Mahmoodi M, Rezaie J (2021) The tumorigenic and therapeutic functions of exosomes in colorectal cancer: opportunity and challenges. Cell Biochem Funct

11. Marin JJG et al (2020) Cellular mechanisms accounting for the refractoriness of colorectal carcinoma to pharmacological treatment. Cancers (Basel) 12(9)

12. Bagheri E, Abnous K, Farzad SA, Taghdisi SM, Ramezani M, Alibolandi M (2020) Targeted doxorubicin-loaded mesenchymal stem cells-derived exosomes as a versatile platform for fighting against colorectal cancer. Life Sci 261:118369

13. Xu Y, Shen L, Li F, Yang J, Wan X, Ouyang M (2019) microRNA16-5p-containing exosomes derived from bone marrow-derived mesenchymal stem cells inhibit proliferation, migration, and invasion, while promoting apoptosis of colorectal cancer cells by downregulating ITGA2. J Cell Physiol 234(11):21380-21394

14. Kara M, Yumrutas O, Ozcan O, Celik OI, Bozgeyik E, Bozgeyik I, Tasdemir S (2015) Differential expressions of cancer-associated 
genes and their regulatory miRNAs in colorectal carcinoma. Gene 567(1):81-86

15. Gao J, Li N, Dong Y, Li S, Xu L, Li X, Li Y, Li Z et al (2015) miR34a-5p suppresses colorectal cancer metastasis and predicts recurrence in patients with stage II/III colorectal cancer. Oncogene 34(31):4142-4152

16. Shi X, Kaller M, Rokavec M, Kirchner T, Horst D, Hermeking H (2020) Characterization of a p53/miR-34a/CSF1R/STAT3 feedback loop in colorectal cancer. Cell Mol Gastroenterol Hepatol 10(2): 391-418

17. Xiao X, Gu Y, Wang $\mathrm{G}$, Chen $\mathrm{S}$ (2019) $c-M y c, R M R P$, and $m i R$ $34 a-5$ form a positive-feedback loop to regulate cell proliferation and apoptosis in multiple myeloma. Int J Biol Macromol 122:526537

18. Yang J, Cao C, Luo D, Lan S, Luo M, Shan H, Ma X, Liu Y et al (2020) Discovery of 4-(3,5-dimethoxy-4-(((4methoxyphenethyl)amino)methyl)phenoxy)- $N$-phenylaniline as a novel c-myc inhibitor against colorectal cancer in vitro and in vivo. Eur J Med Chem 198:112336

19. Lee YJ, Lee EY, Choi BH, Jang H, Myung JK, You HJ (2020) The role of nuclear receptor subfamily 1 group H member 4 (NR1H4) in colon cancer cell survival through the regulation of c-Myc stability. Mol Cell 43(5):459-468

20. Martinez-Useros J et al (2019) UNR/CSDE1 expression is critical to maintain invasive phenotype of colorectal cancer through regulation of c-MYC and epithelial-to-mesenchymal transition. J Clin Med:8(4)

21. Li J, Zhang S, Zou Y, Wu L, Pei M, Jiang Y (2020) miR-145 promotes miR-133b expression through c-myc and DNMT3Amediated methylation in ovarian cancer cells. J Cell Physiol 235(5):4291-4301

22. Huang C, Liu H, Gong XL, Wu L, Wen B (2016) Expression of DNA methyltransferases and target microRNAs in human tissue samples related to sporadic colorectal cancer. Oncol Rep 36(5): 2705-2714

23. Li J, Su X, Dai L, Chen N, Fang C, Dong Z, Fu J, Yu Y et al (2020) Temporal DNA methylation pattern and targeted therapy in colitisassociated cancer. Carcinogenesis 41(2):235-244

24. Liu D et al (2018) Squalene epoxidase drives NAFLD-induced hepatocellular carcinoma and is a pharmaceutical target. Sci Transl Med:10(437)

25. Liu HL, Bao HG, Zheng CL, Teng C, Bai MH (2020) MiR-130a regulating the biological function of colon cancer by targeting inhibition of PTEN. Eur Rev Med Pharmacol Sci 24(4):1786-1793

26. Arabsorkhi Z, Gharib E, Yaghmoorian Khojini J, Farhadieh ME, Nazemalhosseini-Mojarad E, Zali MR (2020) miR-298 plays a pivotal role in colon cancer invasiveness by targeting PTEN. J Cell Physiol 235(5):4335-4350

27. Wang H, Cao F, Li X, Miao H, E J, Xing J, Fu CG (2015) miR-320b suppresses cell proliferation by targeting c-Myc in human colorectal cancer cells. BMC Cancer 15:748

28. Chen M, Xu R, Rai A, Suwakulsiri W, Izumikawa K, Ishikawa H, Greening DW, Takahashi N et al (2019) Distinct shed microvesicle and exosome microRNA signatures reveal diagnostic markers for colorectal cancer. PLoS One 14(1):e0210003

29. Li H, Li F (2018) Exosomes from BM-MSCs increase the population of CSCs via transfer of miR-142-3p. Br J Cancer 119(6):744 755

30. Li YY, Xu QW, Xu PY, Li WM (2020) MSC-derived exosomal $m i R-34 a / c-5 p$ and miR-29b-3p improve intestinal barrier function by targeting the Snail/Claudins signaling pathway. Life Sci 257: 118017

31. Franzen CA et al (2014) Characterization of uptake and internalization of exosomes by bladder cancer cells. Biomed Res Int 2014: 619829
32. Zhang X, Bai J, Yin H, Long L, Zheng Z, Wang Q, Chen F, Yu X et al (2020) Exosomal miR-1255b-5p targets human telomerase reverse transcriptase in colorectal cancer cells to suppress epithelial-to-mesenchymal transition. Mol Oncol 14(10):25892608

33. Wu DM, Wen X, Han XR, Wang S, Wang YJ, Shen M, Fan SH, Zhang ZF et al (2019) Bone marrow mesenchymal stem cellderived exosomal MicroRNA-126-3p inhibits pancreatic cancer development by targeting ADAM9. Mol Ther Nucleic Acids 16:229245

34. Li Z, Yao H, Wang $\mathrm{S}$, Li G, Gu X (2020) CircTADA2A suppresses the progression of colorectal cancer via miR-374a-3p/KLF14 axis. J Exp Clin Cancer Res 39(1):160

35. Zhang Y, Huang W, Yuan Y, Li J, Wu J, Yu J, He Y, Wei Z et al (2020) Long non-coding RNA H19 promotes colorectal cancer metastasis via binding to hnRNPA2B1. J Exp Clin Cancer Res 39(1): 141

36. Zhu L, Zhu Q, Wen H, Huang X, Zheng G (2019) Mutations in GAS5 affect the transformation from benign prostate proliferation to aggressive prostate cancer by affecting the transcription efficiency of GAS5. J Cell Physiol 234(6):8928-8940

37. Teng Y, Zuo X, Hou M, Zhang Y, Li C, Luo W, Li X (2016) A double-negative feedback interaction between microRNA-29b and $D N M T 3 A / 3 B$ contributes to ovarian cancer progression. Cell Physiol Biochem 39(6):2341-2352

38. Zhou $\mathrm{H}$ et al (2017) miR-506 enhances the sensitivity of human colorectal cancer cells to oxaliplatin by suppressing MDR1/P-gp expression. Cell Prolif:50(3)

39. Liao K, Qian Z, Zhang S, Chen B, Li Z, Huang R, Cheng L, Wang $\mathrm{T}$ et al (2020) The LGMN pseudogene promotes tumor progression by acting as a miR-495-3p sponge in glioblastoma. Cancer Lett 490:111-123

40. Yang SS, Li XM, Yang M, Ren XL, Hu JL, Zhu XH, Wang FF, Zeng ZC et al (2017) FMNL2 destabilises COMMD10 to activate NF-kappaB pathway in invasion and metastasis of colorectal cancer. Br J Cancer 117(8):1164-1175

41. Lou $\mathrm{Q}$ et al (2019) miR-448 targets IDO1 and regulates CD8(+) $T$ cell response in human colon cancer. J Immunother Cancer 7(1): 210

42. Chang S, Sun G, Zhang D, Li Q, Qian H (2020) MiR-3622a-3p acts as a tumor suppressor in colorectal cancer by reducing stemness features and EMT through targeting spalt-like transcription factor 4. Cell Death Dis 11(7):592

43. Liang Y, Zhang D, Li L, Xin T, Zhao Y, Ma R, du J (2020) Exosomal microRNA-144 from bone marrow-derived mesenchymal stem cells inhibits the progression of non-small cell lung cancer by targeting CCNE1 and CCNE2. Stem Cell Res Ther 11(1):87

44. Zhu M, Gong Z, Wu Q, Shi X, Su Q, Zhang Y (2020) Sanguinarine suppresses migration and metastasis in colorectal carcinoma associated with the inversion of EMT through the Wnt/beta-catenin signaling. Clin Transl Med 10(1):1-12

45. Saeinasab M, Bahrami AR, González J, Marchese FP, Martinez D, Mowla SJ, Matin MM, Huarte M (2019) SNHG15 is a bifunctional MYC-regulated noncoding locus encoding a lncRNA that promotes cell proliferation, invasion and drug resistance in colorectal cancer by interacting with AIF. J Exp Clin Cancer Res 38(1):172

46. Weis B, Schmidt J, Maamar H, Raj A, Lin H, Tóth C, Riedmann K, Raddatz $\mathrm{G}$ et al (2015) Inhibition of intestinal tumor formation by deletion of the DNA methyltransferase 3a. Oncogene 34(14):18221830

47. Wu Y, Song Y, Xiong Y, Wang X, Xu K, Han B, Bai Y, Li L et al (2017) MicroRNA-21 (Mir-21) Promotes cell growth and invasion by repressing tumor suppressor PTEN in colorectal cancer. Cell Physiol Biochem 43(3):945-958 
48. Qi L, Sun B, Liu Z, Cheng R, Li Y, Zhao X (2014) Wnt3a expression is associated with epithelial-mesenchymal transition and promotes colon cancer progression. J Exp Clin Cancer Res 33:107

49. Wang W, Wang L, Mizokami A, Shi J, Zou C, Dai J, Keller ET, Lu $\mathrm{Y}$ et al (2017) Down-regulation of E-cadherin enhances prostate cancer chemoresistance via Notch signaling. Chin J Cancer 36(1): 35

50. Tauro BJ, Greening DW, Mathias RA, Mathivanan S, Ji H, Simpson RJ (2013) Two distinct populations of exosomes are released from LIM1863 colon carcinoma cell-derived organoids. Mol Cell Proteomics 12(3):587-598

51. Wen XQ, Qian XL, Sun HK, Zheng LL, Zhu WQ, Li TY, Hu JP (2020) MicroRNAs: Multifaceted regulators of colorectal cancer metastasis and clinical applications. Onco Targets Ther 13: 10851-10866

52. Li S, Zhu K, Liu L, Gu J, Niu H, Guo J (2020) lncARSR sponges miR-34a-5p to promote colorectal cancer invasion and metastasis via hexokinase-1-mediated glycolysis. Cancer Sci 111(10):39383952

53. Ma E, Wang Q, Li J, Zhang X, Guo Z, Yang X (2020) LINC01006 facilitates cell proliferation, migration and invasion in prostate cancer through targeting miR-34a-5p to up-regulate DAAMI. Cancer Cell Int 20:515

54. Xu XP et al (2020) miR-34a-5p suppresses the invasion and metastasis of liver cancer by targeting the transcription factor YY1 to mediate MYCT1 upregulation. Acta Histochem 122(6):151576

55. Wang X, Zhao Y, Lu Q, Fei X, Lu C, Li C, Chen H (2020) MiR34a-5p inhibits proliferation, migration, invasion and epithelialmesenchymal transition in esophageal squamous cell carcinoma by targeting LEF1 and inactivation of the hippo-YAP1/TAZ signaling pathway. J Cancer 11(10):3072-3081

56. Wang B, Wu ZH, Lou PY, Chai C, Han SY, Ning JF, Li M (2019) Human bone marrow-derived mesenchymal stem cell-secreted exosomes overexpressing microRNA-34a ameliorate glioblastoma development via down-regulating MYCN. Cell Oncol (Dordr) 42(6):783-799

57. Wan FZ, Chen KH, Sun YC, Chen XC, Liang RB, Chen L, Zhu XD (2020) Exosomes overexpressing miR-34c inhibit malignant behavior and reverse the radioresistance of nasopharyngeal carcinoma. $\mathrm{J}$ Transl Med 18(1):12

58. Li T, Wan Y, Su Z, Li J, Han M, Zhou C (2020) Mesenchymal stem cell-derived exosomal microRNA-3940-5p inhibits colorectal cancer metastasis by targeting integrin alpha6. Dig Dis Sci

59. Xu Y, Liu N, Wei Y, Zhou D, Lin R, Wang X, Shi B (2020) Anticancer effects of miR-124 delivered by BM-MSC derived exosomes on cell proliferation, epithelial mesenchymal transition, and chemotherapy sensitivity of pancreatic cancer cells. Aging (Albany NY) 12(19):19660-19676

60. Aghdam AM, Amiri A, Salarinia R, Masoudifar A, Ghasemi F, Mirzaei H (2019) MicroRNAs as diagnostic, prognostic, and therapeutic biomarkers in prostate cancer. Crit Rev Eukaryot Gene Expr 29(2):127-139

61. Li YJ, du L, Aldana-Masangkay G, Wang X, Urak R, Forman SJ, Rosen ST, Chen Y (2018) Regulation of miR-34b/c-targeted gene expression program by SUMOylation. Nucleic Acids Res 46(14): 7108-7123

62. Munro MJ, Peng L, Wickremesekera SK, Tan ST (2020) Colon adenocarcinoma-derived cells that express induced-pluripotent stem cell markers possess stem cell function. PLoS One 15(5): e0232934

63. Ren L, Zhou T, Wang Y, Wu Y, Xu H, Liu J, Dong X, Yi F et al (2020) RNF8 induces beta-catenin-mediated c-Myc expression and promotes colon cancer proliferation. Int J Biol Sci 16(12):20512062

64. Yang $\mathrm{C}, \mathrm{Lu} \mathrm{W}, \mathrm{He} \mathrm{H}$, Liu $\mathrm{H}$ (2020) Inflammation and DNA methylation-dependent down-regulation of miR-34b-5p mediates c-MYC expression and CRL4(DCAF4) E3 ligase activity in colitis-associated cancer. Am J Pathol 190(3):674-688

65. Ye K, Xu C, Hui T (2019) MiR-34b inhibits the proliferation and promotes apoptosis in colon cancer cells by targeting Wnt/betacatenin signaling pathway. Biosci Rep:39(10)

66. Zhang HL, Wang P, Lu MZ, Zhang SD, Zheng L (2019) $c-M y c$ maintains the self-renewal and chemoresistance properties of colon cancer stem cells. Oncol Lett 17(5):4487-4493

67. Pang Y, Liu J, Li X, Xiao G, Wang H, Yang G, Li Y, Tang SC et al (2018) MYC and DNMT3A-mediated DNA methylation represses microRNA-200b in triple negative breast cancer. J Cell Mol Med 22(12):6262-6274

68. Bhattacharya R, Chatterjee R, Mandal AKA, Mukhopadhyay A, Basu S, Giri AK, Chatterji U, Bhattacharjee P (2020) Theaflavincontaining black tea extract: a potential DNA methyltransferase inhibitor in human colon cancer cells and ehrlich ascites carcinoma-induced solid tumors in mice. Nutr Cancer:1-13

69. Song G, Tian L, Cheng Y, Liu J, Wang K, Li S, Li T (2019) Antitumor activity of sevoflurane in HCC cell line is mediated by miR-29a-induced suppression of Dnmt3a. J Cell Biochem 120(10): 18152-18161

70. Wang J, Luo J, Liu G, Li X (2020) Circular RNA hsa_circ 0008285 inhibits colorectal cancer cell proliferation and migration via the miR-382-5p/PTEN axis. Biochem Biophys Res Commun 527(2):503-510

71. Shi X, Valizadeh A, Mir SM, Asemi Z, Karimian A, Majidina M, Safa A, Yosefi B (2020) miRNA-29a reverses P-glycoproteinmediated drug resistance and inhibits proliferation via upregulation of PTEN in colon cancer cells. Eur J Pharmacol 880: 173138

Publisher's Note Springer Nature remains neutral with regard to jurisdictional claims in published maps and institutional affiliations. 